\title{
Effect of grape seed extract on postprandial oxidative status and metabolic responses in men and women with the metabolic syndrome - randomized, cross-over, placebo-controlled study
}

\section{Indika Edirisinghe ${ }^{1}$, Jody Randolph ${ }^{2}$, Mandeep Cheema ${ }^{2}$, Ravi Tadapaneni ${ }^{1}$, Eunyoung Park $^{1}$, Britt Burton-Freeman ${ }^{1,2}$, Tissa Kappagoda ${ }^{3 *}$}

${ }^{1}$ Center for Nutrition Research, Institute for Food Safety and Health, Illinois Institute of Technology, Bedford Park, IL 60501, USA; ${ }^{2}$ Department of Nutrition, University of California Davis, Davis, CA 95616, USA; ${ }^{3}$ Department of Internal Medicine, University of California Davis, Davis, CA 95616, USA

*Corresponding author: Tissa Kappagoda, PhD, MD, TB 172, Division of Cardiovascular Medicine University of California, Davis, Davis, CA 95616, USA

Submission date: October 17, 2012, Acceptance date: December 18, 2012; Publication date: December 23, 2012

\begin{abstract}
Objective: This investigation was undertaken to determine whether a grape seed extract (GSE) that is rich in mono-, oligo- and poly- meric polyphenols would modify postprandial oxidative stress and inflammation in individuals with the metabolic syndrome (MetS).
\end{abstract}

Background: MetS is known to be associated with impaired glucose tolerance and poor glycemic control. Consumption of a meal high in readily available carbohydrates and fat causes postprandial increases in glycemia and lipidemia and markers of oxidative stress, inflammation and insulin resistance.

Materials/methods: After an overnight fast, twelve subjects with MetS (5 men and 7 women) consumed a breakfast meal high in fat and carbohydrate in a cross-over design. A GSE (300 mg) or placebo capsule was administrated $1 \mathrm{hr}$ before the meal $(-1 \mathrm{hr})$. Changes in plasma insulin, glucose, oxidative stress and inflammatory markers were measured hourly for $6 \mathrm{hr}$.

Results: Plasma hydrophilic oxygen radical absorbance capacity (ORAC) measured as the positive incremental area under the curve ( -1 to $5 \mathrm{hr}$ ) was significantly increased when the meal was preceded by GSE compared with placebo $(\mathrm{P}<0.05)$. Meal-induced increases in oxidized LDL were attenuated with GSE $(\mathrm{P}<0.05)$. Plasma glucose concentrations (area under the curve from -1 to $5 \mathrm{hr}$ ) were also significantly lower when the meal was preceded by GSE $(\mathrm{P}<0.05)$ while the insulin concentrations remained unchanged $(\mathrm{P}>0.05)$. No changes in inflammatory markers were evident. 
Conclusion: These data suggest that GSE enhances postprandial plasma antioxidant status and reduces the glycemic response to a meal, high in fat and carbohydrate in subjects with the MetS.

Key words: Polyphenols, Oxidative stress, Inflammation, ORAC, Oxidized LDL

\section{BACKGROUND:}

Metabolic syndrome (MetS) is a growing medical problem in industrialized countries and is diagnosed when 3 of the following factors are present: abdominal obesity, elevated serum triglycerides, low serum high density lipoprotein (HDL) concentration, elevated blood pressure, and elevated blood glucose [1]. The syndrome is associated with insulin-resistance, impaired glucose control, atherogenic dyslipidemia, oxidative stress, and an enhanced cardiovascular risk $[2,3]$.

Hyperglycemia has been shown to impose cellular oxidative stress through the increased generation of endogenous reactive oxygen species (ROS) particularly in adipose tissue, both in vitro and in vivo. Increased ROS, in turn, promotes inflammation by activating nuclear factor-kappaB (NF- $\mathrm{B}$ ) transcription complex resulting in expression of a number of genes coding for pro-inflammatory cytokines [4,5]. Oxidative stress and inflammation are believed to play critical roles in the pathogenesis of several diseases including atherosclerosis and diabetes [6,7].

We, along with others, have shown that consumption of a meal that is high in readily available carbohydrates and fat results in an increase in markers of oxidative stress and inflammation postprandially [8-13]. These changes are accentuated in obesity, diabetes and individuals with the MetS [14]. Fruits and vegetables, particularly those with higher polyphenolic content, have favorable effects on human health due to their ability to modulate oxidative and inflammatory stress in peripheral tissues [12,13,15]. These effects have also been associated with improvements in insulin sensitivity [16] and postprandial insulin responses [9]. Grape seeds are a concentrated source of polyphenols and have received considerable attention for their antioxidant capacity and biological effects after chronic consumption [17-20]. In the present study, we tested the hypothesis that acute supplementation with grape seed extract (GSE) would attenuate meal-induced alterations in the oxidant and inflammatory status in men and women with the MetS. As a subsidiary investigation we also examined the associated changes in glycemic and insulinemic control.

\section{METHODS:}

\section{Human Clinical trial}

The Human Subjects Research Committee of the University of California, Davis, approved the study. All participants signed a written informed consent form before any study-related procedures were performed; verbal consent was given during the telephone interview prior to answering study screening questions. This study is registered with ClinicalTrials.gov: NCT01190358.

\section{Grape Seed Extract}

The grape seed extract (GSE) used in the study was obtained from Polyphenolic Inc, Madera, 
CA (MegaNatural Gold@). Total phenols in the extract was 94.3 gallic acid equivalents (wt/wt) as assessed by the Folin \& Ciocalteu method [21]. The moisture content was $4.1 \%$. The detailed polyphenolic composition as assessed using LC-MS/MS is given in Table 1. This extract has received Generally Recognized as Safe (GRAS) status from Food and Drug Administration (No. GRN 000125).

Table 1: polyphenolic composition of the Grape Seed Extract (GSE- MegaNatural GoldC)

\begin{tabular}{|l|l|}
\hline Name of compound & \% Composition \\
\hline Gallic Acid & 5.7 \\
\hline Catechin & 6.7 \\
\hline Epicatechin & 4.8 \\
\hline Dimer B & 34.4 \\
\hline Polymer & 46.1 \\
\hline
\end{tabular}

\section{Subjects}

Twelve subjects ( $\mathrm{n}=12,7$ females and 5 males) identified with MetS according to the NCEP III guidelines [1] were recruited through newspapers, posters, and direct contact in the Davis and Sacramento area of northern California. All 12 subjects completed the study. Eligible subjects were required to be non-smokers and in generally good health. Individuals, a) with clinical evidence and/or history of cardiovascular, respiratory, renal, gastrointestinal, metabolic or hepatic disease/conditions, b) who used medications that may interfere with study endpoints (i.e. anti-inflammatory drugs, lipid lowering medications, antioxidant supplements), c) who had unusual dietary habits (e.g., pica), d) who were actively losing weight and e) who were addicted to drugs and/or alcohol were not eligible for participation.

\section{Study Design and Treatments}

This study was a single-center, randomized, cross-over, 2-arm, placebo-controlled, $5 \mathrm{hr}$ postprandial trial conducted at the Clinical and Translational Science Center (CTSC) at the University of California Davis - affiliated Northern CA Veteran's Affairs (VA) Medical Center in Mather, CA. Enrolled participants were required to limit consumption of foods containing polyphenolic products throughout the study period starting 1 week prior to the first study visit; all other aspects of diet and physical activity remained unchanged.

Subjects were randomized to receive one of two capsules (GSE or Placebo) along with a high fat-carbohydrate breakfast meal in a randomly selected sequence. Each subject received both of the treatments $\sim 1$ week apart and served as his/her own control. The high fatcarbohydrate breakfast meal was prepared to provide approximately $670 \mathrm{kcal}$ with $40 \%$ of energy from fat (Table 2). Test meals were prepared in the metabolic kitchen at the CTSC with strict adherence to food and safety standards. 
Table 2: Composition of the test meal

\begin{tabular}{|c|c|}
\hline Bagel $^{\#}$ & $110 \mathrm{~g}$ \\
\hline Cream cheese & $14 \mathrm{~g}$ \\
\hline Margarine $^{\$}$ & $5 \mathrm{~g}$ \\
\hline Egg & $50 \mathrm{~g}$ \\
\hline Cantaloupe & $85 \mathrm{~g}$ \\
\hline Whole milk & $240 \mathrm{ml}$ \\
\hline Calorie content & \begin{tabular}{|l}
$\sim 670 \mathrm{Kcal}$ \\
\end{tabular} \\
\hline Calories from fat & $40 \%$ \\
\hline
\end{tabular}

*All ingredients were purchased from local supermarkets.

\# Bagel was toasted. $\$$ Margarine was used to scramble the egg.

\section{Postprandial Testing Protocol}

All procedures were identical during both study visits with the exception of the capsule (GSE or Placebo) consumed. Postprandial testing was conducted according to standardized protocols we described previously (8-10). Subjects arrived at the CTSC in the morning after an overnight fast on two separate occasions; study visits were between 7 and 10 days apart. On study visit mornings, a Registered Nurse placed an indwelling catheter in the antecubital vein of the nondominant arm of subjects. Immediately following the initial blood draw (-1 hr), subjects were orally administered a GSE or Placebo capsule with water. One hr later, a pre-meal blood sample was drawn $(0 \mathrm{hr})$ and then subjects ate the high fat-carbohydrate breakfast meal comprised of common breakfast foods (Table 2) consumed within 20 minutes. Blood was collected hourly for $5 \mathrm{hr}$ after meal consumption (Figure 1, Study schema) and processed for subsequent analysis of plasma lipids, glucose, insulin, oxidized LDL (OxLDL), interleukin-6 (IL-6) and tumor necrosis factor-alpha (TNF- $\alpha$ ). Three day food records ( 1 set per week) were maintained and reviewed by a dietitian with subjects for compliance to limiting polyphenolic intake throughout the study.

\section{Figure 1}

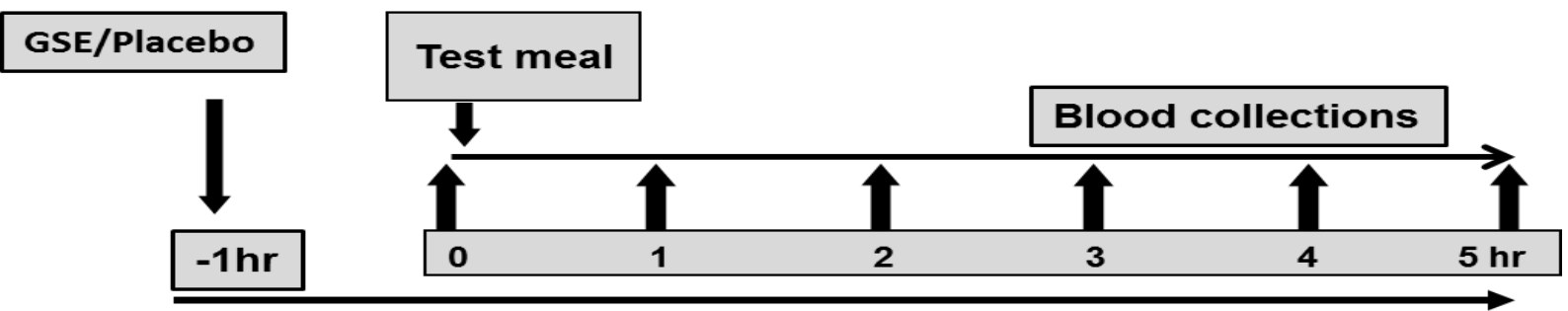

Figure 1: Study schema. Schematic of the blood sampling at each Postprandial Test Day Visit.

\section{Analytical Methods}

Several analytical methods were used to assess the effect of GSE consumption on fasting and postprandial concentrations of plasma lipids, glucose, insulin, OxLDL, IL-6 and TNF- $\alpha$. Lipids and glucose were measured using standardized enzyme-based assay kits (Randox, Antrim, UK) 
on the Randox Daytona Auto Clinical Analyzer (Randox, Antrim, UK). Insulin was measured using the AlphaLISA method (Perkin Elmer, Waltham, MA). Measurement of OxLDL was done using ELISA assay kits (Mercodia Inc., Winston Salem, NC) and IL-6 and TNF- $\alpha$ were measured by highly-sensitive ELISA assays (R\&D Systems, Minneapolis, MN). All assay protocols were performed according to the manufacturers' instructions and appropriate quality controls were used as applicable. Oxygen radical absorbance capacity (ORAC) assay was performed according to the method described by Prior et al [22].

\section{Statistical Analysis}

Postprandial changes in glucose, triglycerides, cholesterol, insulin and ORAC in response to GSE or placebo with the high fat-carbohydrate meals were compared based on total area under the curve (AUC) from $-1 \mathrm{hr}$ to $5 \mathrm{hr}$. When baseline (fasting) values were different, as observed with OxLDL, ORAC, IL-6 and TNF- $\alpha$, data were normalized to subjects own baseline prior to AUC computation and analysis. Analyses of AUC for OxLDL, ORAC, IL- 6 and TNF- $\alpha$ are based on the positive incremental AUC (negative values after baseline corrections were omitted in the analysis). AUC was calculated using the trapezoidal method. Treatment associated differences over time and at each time-point was analyzed via PROC MIXED using PC-SAS (version 9.2; SAS Institute Inc, Cary, NC). The level used to determine statistical significance was $\mathrm{p}<0.05$.

\section{RESULTS:}

\section{Subject Characteristics}

A total of twelve subjects completed the study ( $n=7$ females, $n=5$ males). The age and BMI of the subjects were $45 \pm 15 \mathrm{y}$ and $36 \pm 7 \mathrm{~kg} / \mathrm{m}^{2}$, respectively (Mean $\pm \mathrm{SD}$ ). Baseline $(-1 \mathrm{hr}$ ) metabolic panel is listed in Table 3 .

Table 3: Baseline Characteristics of study subjects $(n=12)$

\begin{tabular}{|l|l|}
\hline Male/Female $(\mathrm{N})$ & $5 / 7$ \\
\hline BMI $\left(\mathrm{kg} / \mathrm{m}^{2}\right)$ & $35.9 \pm 6.7$ \\
\hline Age $(\mathrm{Y})$ & $45 \pm 15$ \\
\hline & \\
\hline Base line metabolic Panel & \\
\hline Glucose $(\mathrm{mg} / \mathrm{dL})$ & $104.5 \pm 22.8$ \\
\hline Insulin $(\mu \mathrm{IU} / \mathrm{mL})$ & $25.0 \pm 7.2$ \\
\hline HOMA -IR & $3.3 \pm 1.1$ \\
\hline Triglycerides $(\mathrm{mg} / \mathrm{dL})$ & $156.0 \pm 38.9$ \\
\hline Total Cholesterol $(\mathrm{mg} / \mathrm{dL})$ & $197.1 \pm 56.2$ \\
\hline LDL Cholesterol $(\mathrm{mg} / \mathrm{dL})$ & $122.6 \pm 45.6$ \\
\hline HDL Cholesterol $(\mathrm{mg} / \mathrm{dL})$ & $43.7 \pm 11.6$ \\
\hline Systolic Blood Pressure $(\mathrm{mmHg})$ & $132.5 \pm 11.0$ \\
\hline Diastolic Blood Pressure $(\mathrm{mmHg})$ & $78.7 \pm 8.8$ \\
\hline
\end{tabular}




\section{Postprandial responses}

Oxidative stress markers were assessed using plasma and plasma ORAC (hydrophilic and lipophilic) and OxLDL assays. Plasma ORAC values were assessed in hydrophilic and lipophilic fractions separately (Figure 2 A \& B). Peak plasma hydrophilic and lipophilic ORAC values were observed at $1 \mathrm{hr}$ after the meal in both GSE and placebo treated groups, and were significantly different compared to their respective baseline values $(-1 \mathrm{hr}, P<0.05)$. The plasma hydrophilic and lipophilic ORAC values at baseline $(-1 \mathrm{hr})$ and $5 \mathrm{hr}$ after the meal were not significantly different in response to GSE or placebo treatments $(P>0.05)$. Analysis of the positive incremental AUC response (-1 to $5 \mathrm{hr}$ ) revealed significantly increased hydrophilic ORAC values with GSE groups compared to placebo (GSE- hydrophilic positive incremental AUC $3064.3 \pm 570.3$ vs. positive incremental AUC Placebo $1034.7 \pm 462.6 \mu \mathrm{mol}$.hr/L Trolox equivalents, $P<0.05)$. However, analysis of the positive incremental AUC response in lipophilic ORAC values were not significantly different in GSE Vs Placebo group $(P>0.05)$.

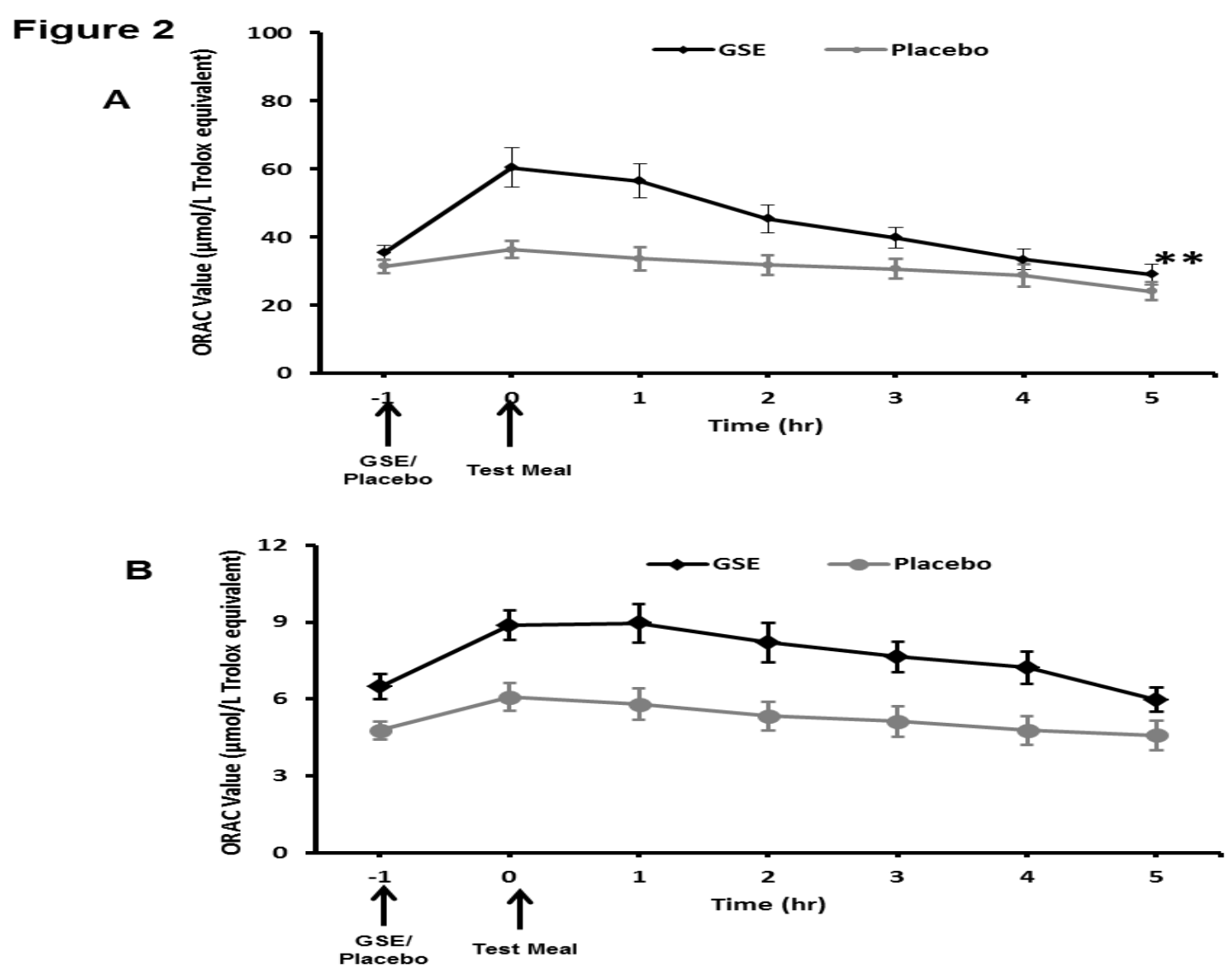

Figure 2: Postprandial plasma Oxygen Radical Absorbance Capacity (ORAC) values.

(A) Hydrophilic ORAC values and (B) lipophilic ORAC values. Values represent mean plasma ORAC values with their standard errors by vertical bars at respective time points before and after a high fat-carbohydrate breakfast meal with the grape seed extract (GSE) or the placebo. Post prandial ORAC values in the hydrophilic phase as assessed by positive incremental area under the curve was significantly increased after the meal with the GSE compared with the placebo $(* * \mathrm{P}<0.01, \mathrm{n}=12)$. 
Changes in glucose, lipids, insulin and biomarkers of oxidative stress and inflammation were measured over the $6 \mathrm{hr}$ experimental period at defined intervals after capsule consumption (Figure 1). Responses after the GSE and placebo along with test meals were compared as total AUC or positive incremental AUC from $-1 \mathrm{hr}$ to $5 \mathrm{hr}$. Total AUC for triglyceride and cholesterol responses were not significantly different between groups ( $\mathrm{P}>0.05$, data not shown).

Postprandial OxLDL concentrations were significantly increased at $5 \mathrm{hr}$ after the breakfast meal compared to fasting values $(-1 \mathrm{hr})$ in the placebo group $(P<0.05)$, whereas in the GSE group, OxLDL concentrations were significantly reduced at $5 \mathrm{hr}$ after the breakfast meal compared to fasting concentrations (-1 hr) (Figure 3). Significant changes were not observed with IL-6 and TNF- $\alpha$ between GSE and placebo groups (Figure 4 A \& B)

\section{Figure 3}

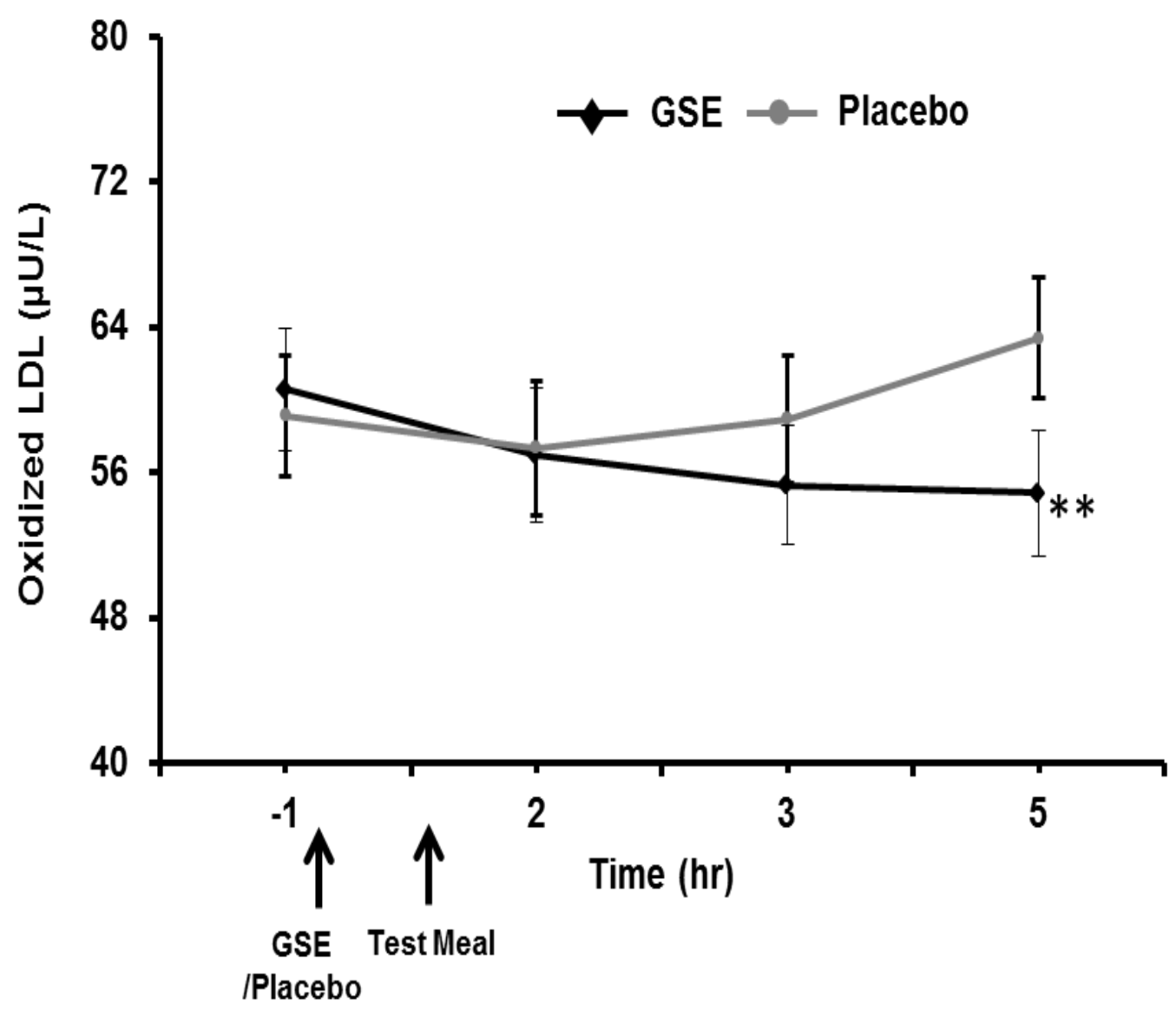

Figure 3: Postprandial plasma oxidized LDL (OxLDL) concentrations. Values represent mean plasma Ox-LDL concentrations with their standard errors by vertical bars at respective time points before and after a high fat-carbohydrate breakfast meal with the grape seed extract (GSE) or the placebo. Post prandial OxLDL concentrations at $5 \mathrm{hr}$ time point is significantly decreased in GSE compared with the placebo $(* * \mathrm{P}<0.01, \mathrm{n}=12)$. 


\section{Figure 4}

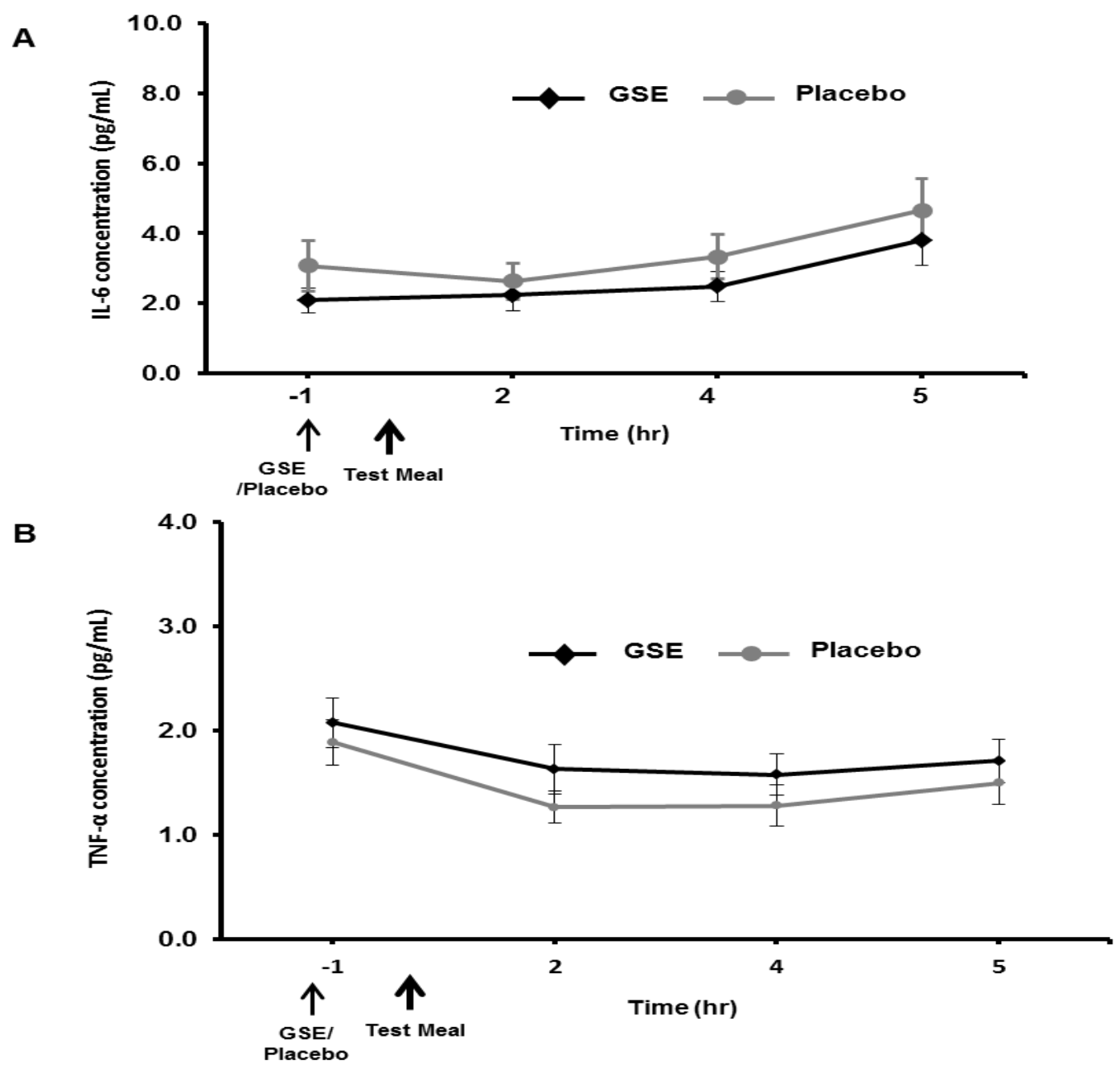

Figure 4: Postprandial plasma inflammatory markers. (A): Interlukin-6 (IL-6) (B): Tumor necrotic factor- $\alpha$ (TNF- $\alpha$ ). Values represent mean plasma IL- 6 or TNF- $\alpha$ concentrations with their standard errors by vertical bars at respective time points before and after a high -fatcarbohydrate breakfast meal with the grape seed extract (GSE) or the placebo. Postprandial plasma IL- 6 or TNF- $\alpha$ concentrations as assessed by positive area under the curve were not significantly different after the meal with the GSE compared with the placebo $(\mathrm{P}>0.05, \mathrm{n}=12)$.

The postprandial glucose response after consumption of the meal preceded by the placebo or GSE capsule (at $-1 \mathrm{hr}$ ) is shown in Figure 5. Between $-1 \mathrm{hr}$ and $0 \mathrm{hr}$ (just before meal consumption), glucose concentrations did not significantly differ $(P>0.05)$. After the meal, glucose concentrations peaked at approximately $1 \mathrm{hr}$ after both treatments and the values at $1 \mathrm{hr}$ did not significantly differ between two groups $(P>0.05)$. Analysis of total AUC revealed significantly lower glucose over the $6 \mathrm{hr}$ experimental period with GSE compared to placebo (GSE total AUC $667.4 \pm 63.6$ vs. total AUC placebo $691.0 \pm 59.1 \mathrm{mg} / \mathrm{dL} * \mathrm{hr}, P<0.05$ ). Peak plasma insulin concentrations were also observed at approximately $1 \mathrm{hr}$ after meal consumption for both treatments. The insulin responses in the GSE treated group compared to the placebo 
group were not significantly different (Total GSE AUC $251.7 \pm 96.2$ vs. placebo $262.8 \pm 82$ $\mu \mathrm{IU} / \mathrm{L} * \mathrm{hr}, P>0.05$, Figure 6).

Figure 5

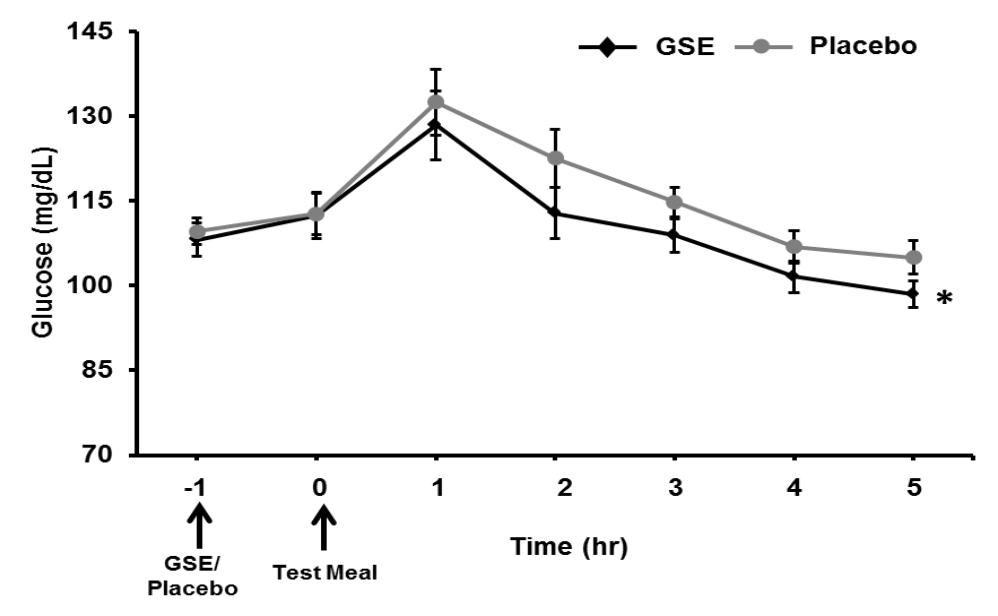

Figure 5: Postprandial plasma glucose concentrations. Values represent mean plasma glucose concentrations with their standard errors by vertical bars at respective time points before and after a high fat-carbohydrate breakfast meal with the grape seed extract (GSE) or the placebo. Postprandial glucose concentrations as assessed by total area under the curve was significantly lower after the meal with the GSE compared with the placebo $(* \mathrm{P}<0.05, \mathrm{n}=12$ ).

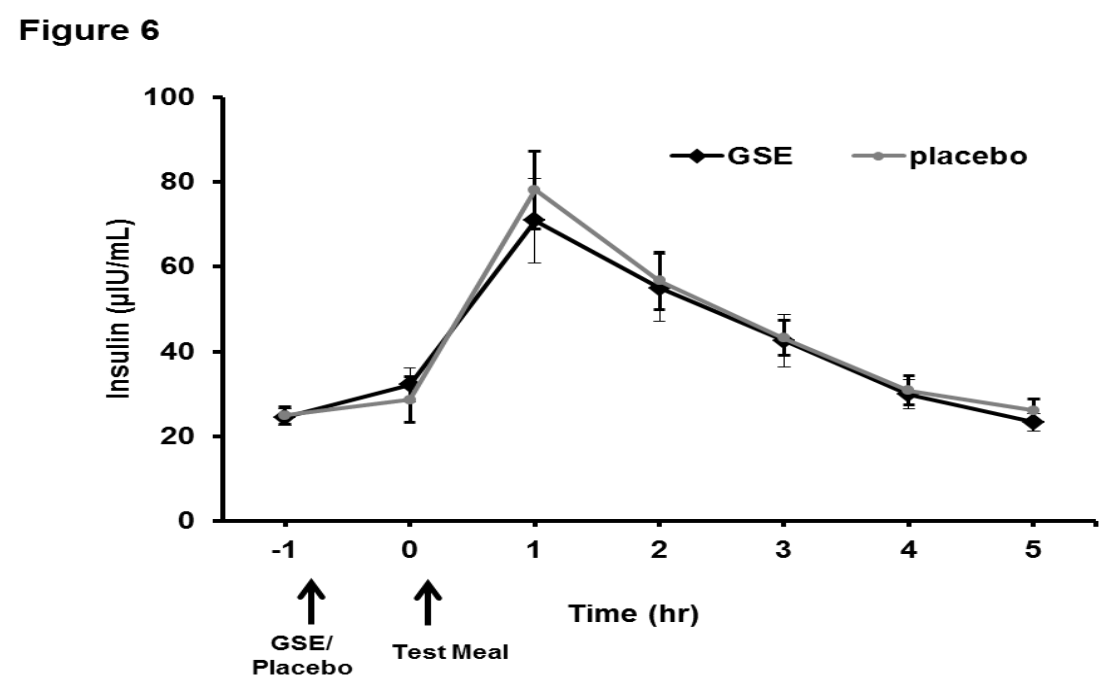

Figure 6: Postprandial plasma insulin concentrations. Values represent mean plasma insulin concentrations with their standard errors by vertical bars at respective time points before and after a high fat-carbohydrate breakfast meal with the grape seed extract (GSE) or the placebo. Postprandial glucose concentrations as assessed by total area under the curve was not significantly different after the meal with the GSE compared with the placebo $(\mathrm{P}>0.05, \mathrm{n}=12)$. 


\section{DISCUSSION:}

Postprandial oxidative stress resulting from consumption of meals high in readily available carbohydrate and fat, which are typical of Westernized eating patterns, has been proposed as a major factor in the pathogenesis of chronic diseases, such as diabetes and cardiovascular disease [23]. Our previous findings that consumption of such a meal results in a postprandial increase in markers of oxidative stress, inflammation and insulin response are consistent with this view [810]. In the present study, we tested the hypothesis that acute supplementation with GSE before a meal would attenuate the postprandial oxidant and inflammatory changes in people with the MetS. The major findings of this study were that GSE i) enhanced the postprandial antioxidant capacity of the plasma and suppressed the increases in oxidized LDL and ii) reduced postprandial glucose concentrations compared to placebo in men and women with MetS.

GSE is rich in polyphenolic compounds and has a unique profile of complex polymers and simple phenolic compounds. Although the antioxidant activity of the GSE is well documented in vitro [20], its activity in vivo is less well defined. One reason for this apparent discrepancy is that polyphenolic compounds are extensively conjugated in the body and only a minor fraction remains unconjugated, thereby making it difficult to link biological effects to structure [24,25]. This is particularly evident in the case of grape derived products which are monomeric and are often altered during absorption by gut microbiota. Thus bioavailability may be underestimated due to poor detection and identification of metabolites [26]. Nonetheless, we have demonstrated previously that flavon-3-ol catechins from GSE are absorbed into the blood and the maximum plasma concentrations $\left(\mathrm{C}_{\max }\right)$ occurs between 90-120 min after ingestion [27]. In the present study, peak plasma glucose and triglyceride concentrations were observed at 30 and $180 \mathrm{~min}$, respectively after meals [8-10]. Thus, GSE administrated $1 \mathrm{hr}$ before the test meal, allowed for elevated concentrations of GSE compounds/metabolites and peak glucose and triglyceride concentrations to overlap maximizing the potential effects of GSE during the postprandial period.

In the present study, we have shown that GSE modulates the oxidative stress associated with a single high fat meal in people with the MetS. It is not known whether these effects resulting from a single dose would influence responses following other meals consumed during a day. These responses were associated with decreased postprandial glucose concentrations and an unchanged plasma insulin profile. One interpretation of these findings is that the GSE altered glucose absorption; however, if this were the case, we would have expected differences between treatments in the postprandial insulin response, which we did not observe.

Insulin signaling is impaired under conditions of oxidative stress [28] and polyphenols have been shown to reverse oxidative stress-induced impairments in insulin signaling [29]. Thus our findings suggest that the effects of GSE on decreased plasma glucose are mediated through improved insulin economy, possibly through modulation of cellular redox status improving insulin signaling. In-vitro cell culture studies show that oxidative stress increases serine (Ser307) phosphorylation in Insulin Receptor Substrate 1 (IRS-1) protein molecules. Serine phosphorylation in IRS-1 is known to inhibit insulin-mediated downstream signaling resulting in impaired glucose uptake [30,31]. Preliminary in-vitro studies performed in our laboratory has shown that treatment of skeletal muscle cells with a strawberry extract under oxidative stress conditions decreases IRS-1, Ser-307 phosphorylation and increases IRS-1, Tyr phosphorylation, 
the stimulatory mechanism for insulin-mediated downstream signaling [32]. In overweight men and women, consumption of a strawberry beverage with a high carbohydrate-moderate fat meal significantly reduced the postprandial insulin response compared to when the meal was consumed with a placebo beverage, while the glucose response did not differ between treatments [9]. Stull et al, 2010 reported increased insulin sensitivity in obese, non-diabetic, and insulinresistant subjects after 8 weeks blueberry supplementation using the hyperinsulinemic euglycemic clamp procedure [16] and Brasnyó et al showed that resveratrol, a stilbene polyphenol found in red wine, improved insulin sensitivity in human subjects with type II diabetes [33]. Taken collectively, the results of the current study along with the findings from animal studies $[18,19]$ suggests that GSE could improve insulin resistance. However, future studies using conventional insulin clamp procedures are required to confirm this effect [16].

Postprandial inflammatory markers as assessed by plasma IL-6 and TNF- $\alpha$ concentration were not affected by GSE. Our previous work with strawberries suggests that markers of the inflammatory response are not significantly elevated in plasma until much later in the postprandial period (6 hr) [9]. Therefore, it is likely that the present study may have been too short to detect significant changes in inflammatory cytokines in this population of MetS subjects in response to the meal and treatments.

\section{Limitations of the study}

This study focused on the effect of single meal (breakfast) that was high in fat and carbohydrates. The findings do not address the issue of whether a single dose of this extract administered before breakfast would influence the effects of other similar meals consumed during the rest of the day. Multiple meal studies would provide answers to these questions and are underway in our laboratory. Likewise, the data cannot be extrapolated to long-term effects on glucose control or improvements in insulin sensitivity. Further studies are required to confirm our findings and test the ability of GSE to prevent the conversion of those with the metabolic syndrome into overt diabetes. Finally, our findings are only suggestive of an improvement in insulin resistance and a definitive estimate would require a study that includes an insulin clamp procedure [34]. Previous studies have shown that bioactive compounds in blueberry improved insulin sensitivity as measured using the insulin clamp procedure [16].

\section{CONCLUSIONS:}

Administration of GSE one hr before breakfast enhanced antioxidant status of people with MetS and was associated with decreased postprandial glycemia, suggesting enhanced insulin economy. The clinical implications of our findings require additional research; however, these findings support a growing body of research that suggests consuming polyphenol rich foods/extracts with or within an hr of a meal can minimize the unfavorable perturbations in postprandial redox balance and metabolism induced by modern day eating and lifestyle patterns.

Abbreviations: MetS-Metabolic syndrome, HDL- high density lipoprotein, ROS -reactive oxygen species, NF- $\kappa \mathrm{B}$-nuclear factor-kappaB, GSE -grape seed extract, CTSC-Clinical and Translational Science Center, OxLDL- oxidized LDL, IL-6-interleukin-6, TNF- $\alpha$ - tumor 
necrosis factor-alpha, ORAC -Oxygen radical absorbance capacity, AUC -area under the curve, $\mathrm{C}_{\max }$-maximum plasma concentrations, IRS-1-Insulin Receptor Substrate 1.

Authors Disclosure: The project was funded by polyphenolic Inc, Madera, CA.

\section{Competing Interests: None}

Authors' Contributions: Indika Edirisinghe: helped with design of project, supervised biochemical aspects of study, data analysis, and write-up; Jody Randolph: Study coordinator; Mandeep Cheema: assisted with study coordination; Ravi Tadapaneni: assisted with biochemical analysis; Eunyoung Park: Statistical analysis; Britt Burton-Freeman: Study design, data analysis and write up; Tissa Kappagoda: Principal Investigator, Study design, Clinical supervision.

Acknowledgments: The project was funded by polyphenolic Inc, Madera, CA.

\section{REFERENCES:}

1. Executive Summary of The Third Report of The National Cholesterol Education Program (NCEP) Expert Panel on Detection, Evaluation, And Treatment of High Blood Cholesterol In Adults (Adult Treatment Panel III). JAMA. United States, 2001:2486-97.

2. Hansel B, Giral P, Nobecourt E et al. Metabolic syndrome is associated with elevated oxidative stress and dysfunctional dense high-density lipoprotein particles displaying impaired antioxidative activity. J Clin Endocrinol Metab. United States, 2004:4963-71.

3. Furukawa S, Fujita T, Shimabukuro M et al. Increased oxidative stress in obesity and its impact on metabolic syndrome. J Clin Invest 2004;114:1752-61.

4. Lin Y, Berg AH, Iyengar P et al. The hyperglycemia-induced inflammatory response in adipocytes: the role of reactive oxygen species. J Biol Chem. United States, 2005:461726.

5. Espiritu DJ, Huang ZH, Zhao Y, Mazzone T. Hyperglycemia and advanced glycosylation end products suppress adipocyte apoE expression: implications for adipocyte triglyceride metabolism. Am J Physiol Endocrinol Metab. United States, 2010:E615-23.

6. Lankin VZ, Lisina MO, Arzamastseva NE et al. Oxidative stress in atherosclerosis and diabetes. Bull Exp Biol Med. United States, 2005:41-3.

7. Pennathur S, Heinecke JW. Mechanisms for oxidative stress in diabetic cardiovascular disease. Antioxid Redox Signal 2007;9:955-69.

8. Burton-Freeman B, Talbot J, Park E, Krishnankutty S, Edirisinghe I. Protective activity of processed tomato products on postprandial oxidation and inflammation: a clinical trial in healthy weight men and women. Mol Nutr Food Res 2012;56:622-31.

9. Edirisinghe I, Banaszewski K, Cappozzo J et al. Strawberry anthocyanin and its association with postprandial inflammation and insulin. Br J Nutr. England, 2011:913-22.

10. Burton-Freeman B, Linares A, Hyson D, Kappagoda T. Strawberry modulates LDL oxidation and postprandial lipemia in response to high-fat meal in overweight hyperlipidemic men and women. J Am Coll Nutr 2010;29:46-54. 
11. Hyson DA, Paglieroni TG, Wun T, Rutledge JC. Postprandial lipemia is associated with platelet and monocyte activation and increased monocyte cytokine expression in normolipemic men. Clin Appl Thromb Hemost 2002;8:147-55.

12. Blanco-Colio LM, Valderrama M, Alvarez-Sala LA et al. Red wine intake prevents nuclear factor-kappaB activation in peripheral blood mononuclear cells of healthy volunteers during postprandial lipemia. Circulation 2000;102:1020-6.

13. Ghanim H, Sia CL, Upadhyay $M$ et al. Orange juice neutralizes the proinflammatory effect of a high-fat, high-carbohydrate meal and prevents endotoxin increase and Toll-like receptor expression. Am J Clin Nutr. United States, 2010:940-9.

14. Perez-Martinez P, Garcia-Quintana JM, Yubero-Serrano EM et al. Postprandial oxidative stress is modified by dietary fat: evidence from a human intervention study. Clin Sci (Lond). England, 2010:251-61.

15. Rahman I, Biswas SK, Kirkham PA. Regulation of inflammation and redox signaling by dietary polyphenols. Biochem Pharmacol. England, 2006:1439-52.

16. Stull AJ, Cash KC, Johnson WD, Champagne CM, Cefalu WT. Bioactives in blueberries improve insulin sensitivity in obese, insulin-resistant men and women. J Nutr. United States, 2010:1764-8.

17. Leifert WR, Abeywardena MY. Cardioprotective actions of grape polyphenols. Nutr Res. United States, 2008:729-37.

18. Chis IC, Ungureanu MI, Marton A et al. Antioxidant effects of a grape seed extract in a rat model of diabetes mellitus. Diab Vasc Dis Res. England, 2009:200-4.

19. Meeprom A, Sompong W, Suwannaphet W, Yibchok-anun S, Adisakwattana S. Grape seed extract supplementation prevents high-fructose diet-induced insulin resistance in rats by improving insulin and adiponectin signalling pathways. $\mathrm{Br} \mathrm{J}$ Nutr. England, 2011:1173-81.

20. Kim Y, Choi Y, Ham H, Jeong HS, Lee J. Antioxidant and cytoprotective effects of oligomeric and polymeric procyanidin fractions from defatted grape seed in PC12 cells. J Med Food 2012;15:490-4.

21. Slinkard K S, VL. Total Phenol Analysis: Automation and Comparison with Manual Methods. Am J Enol Vitic 1977;28:49-55.

22. Prior RL, Hoang H, Gu L et al. Assays for hydrophilic and lipophilic antioxidant capacity (oxygen radical absorbance capacity (ORAC(FL))) of plasma and other biological and food samples. J Agric Food Chem 2003;51:3273-9.

23. Sies H, Stahl W, Sevanian A. Nutritional, dietary and postprandial oxidative stress. J Nutr. United States, 2005:969-72.

24. Del Rio D, Costa LG, Lean ME, Crozier A. Polyphenols and health: what compounds are involved? Nutr Metab Cardiovasc Dis. Germany: 2009 Elsevier B.V, 2010:1-6.

25. Galvano F, La Fauci L, Vitaglione P, Fogliano V, Vanella L, Felgines C. Bioavailability, antioxidant and biological properties of the natural free-radical scavengers cyanidin and related glycosides. Ann Ist Super Sanita 2007;43:382-93. 
26. Laparra JM, Sanz Y. Interactions of gut microbiota with functional food components and nutraceuticals. Pharmacol Res. England: 2009 Elsevier Ltd, 2010:219-25.

27. Sivaprakasapillai B, Edirisinghe I, Randolph J, Steinberg F, Kappagoda T. Effect of grape seed extract on blood pressure in subjects with the metabolic syndrome. Metabolism. United States, 2009:1743-6.

28. Rudich A, Tirosh A, Potashnik R, Hemi R, Kanety H, Bashan N. Prolonged oxidative stress impairs insulin-induced GLUT4 translocation in 3T3-L1 adipocytes. Diabetes 1998;47:1562-9.

29. Hininger-Favier I, Benaraba R, Coves S, Anderson RA, Roussel AM. Green tea extract decreases oxidative stress and improves insulin sensitivity in an animal model of insulin resistance, the fructose-fed rat. J Am Coll Nutr. United States, 2009:355-61.

30. Werner ED, Lee J, Hansen L, Yuan M, Shoelson SE. Insulin resistance due to phosphorylation of insulin receptor substrate-1 at serine 302. J Biol Chem. United States, 2004:35298-305.

31. Tanti JF, Gual P, Gremeaux T, Gonzalez T, Barres R, Le Marchand-Brustel Y. Alteration in insulin action: role of IRS-1 serine phosphorylation in the retroregulation of insulin signalling. Ann Endocrinol (Paris). France, 2004:43-8.

32. Sandhya K, Tadapaneni R, Banaszewski K, Cappozzo J, Edirisinghe I, Burton-Freeman B. Strawberry extract attenuates oxidative stress-induced impaired insulin signaling in vitro in Human Skeletal Muscle Cells. The FASEB Journal 2010;24:541.13.

33. Brasnyo P, Molnar GA, Mohas $\mathrm{M}$ et al. Resveratrol improves insulin sensitivity, reduces oxidative stress and activates the Akt pathway in type 2 diabetic patients. $\mathrm{Br} \mathrm{J}$ Nutr. England, 2011:383-9.

34. Trout KK, Homko C, Tkacs NC. Methods of measuring insulin sensitivity. Biol Res Nurs 2007;8:305-18. 